\title{
Steady-State Coherent Transfer by Adiabatic Passage
}

\author{
Jan Huneke, Gloria Platero, and Sigmund Kohler \\ Instituto de Ciencia de Materiales, CSIC, Cantoblanco, E-28049 Madrid, Spain
}

(Dated: June 14, 2022)

\begin{abstract}
We propose steady-state electron transport based on coherent transfer by adiabatic passage (CTAP) in a linearly arranged triple quantum dot with leads attached to the outer dots. Its main feature is repeated steering of single electrons from the first dot to the last dot without relevant occupation of the middle dot. The coupling to leads enables a steady-state current, whose shot noise is significantly suppressed provided that the CTAP protocol performs properly. This represents an indication for the direct transfer between spatially separated dots and, thus, may resolve the problem of finding experimental evidence for the non-occupation of the middle dot.
\end{abstract}

PACS numbers: 05.60.Gg, 73.63.Kv, 73.23.Hk

The possibility of finding particles in delocalized states is a most intriguing feature of quantum systems. It gives rise to phenomena such as tunneling which is intrinsically quantum mechanical and beyond everyday experience. A particular tunnel effect is the coherent transfer by adiabatic passage (CTAP) [1 of an electron in a linearly arranged triple quantum dot 2, 3. It is based on the control of the inter-dot tunnelings, such that an electron is guided from the first dot to the last dot without populating the middle dot. While being closely related to stimulated Raman adiabatic passage [4, 5, CTAP possesses the appealing feature of dealing with spatially separated states.

The non-occupation of the middle dot can be theoretically predicted straightforwardly by solving the Schrödinger equation or, in the presence of decoherence, an appropriate quantum master equation. By contrast, its direct experimental proof is less obvious, because such position measurement unavoidably acts back on the position. Thus, such measurement would influence the effect that it should substantiate. For example, coupling the middle dot to a charge detector [6, 7] impacts significantly on the dot populations [8, since it causes decoherence to which CTAP is quite sensitive [9. Consequently, as a matter of principle, direct observation of the dot populations is of limited use and, thus, one may have to resort to an indirect verification.

In this work, we propose a generalization of CTAP that circumvents this difficulty and predicts an experimental fingerprint for the non-occupation of the middle dot. It is based on a modified setup with electron reservoirs attached to the first and the last quantum dot (see Fig. 1), such that a current may flow. We demonstrate that the suggested steady-state CTAP protocol establishes a rather regular electron transport with suppressed shot noise indicating its proper course. In turn, if the middle dot is significantly occupied, transport becomes more noisy. Our analysis of the transport process is based on a numerical propagation of a time-dependent quantum master equation, which needs to be augmented in order to compute current noise [10, 11].
Model and master equation. - The setup sketched in Fig. 1 is described by the Hamiltonian $H(t)=H_{\mathrm{TQD}}(t)+$ $H_{\text {dot-leads }}+H_{L}+H_{R}$, where (in units with $\hbar=1$ )

$$
H_{\mathrm{TQD}}(t)=\Omega_{12}(t) c_{1}^{\dagger} c_{2}+\Omega_{23}(t) c_{2}^{\dagger} c_{3}+\text { H.c. }
$$

refers to the triple quantum dot with vanishing onsite energies. We assume strong Coulomb repulsion such that the triple dot can be occupied with at most one electron. Hence the electron spin has no relevant influence and will be ignored. By applying appropriate gate voltages to the inter-dot barriers, the tunnel matrix elements between neighboring dots, $\Omega_{12}(t)$ and $\Omega_{23}(t)$, are controlled such that they assume the sequence of double Gauss pulses

$$
\Omega_{12 / 23}(t)=\sum_{n=0}^{\infty} \Omega_{\max } \exp \left[-\frac{\left(t \pm \Delta t / 2-n T-t_{0}\right)^{2}}{2 \sigma^{2}}\right]
$$

with amplitude $\Omega_{\max }$, width $2 \sigma$, delay time $\Delta t$, and repetition time $T$ as is depicted in Fig. 2(a). This implies the time periodicity $H(t)=H(t+T)$ for $t>t_{0}$ with some time-offest $t_{0}$.

Ideally, one would like to modify the control parameters $\Omega_{i j}$ adiabatically slowly to ensure ideal accomplishment of the protocol. This however is not possible, because a lower limit to the CTAP duration is set by decoherence, which may stem from substrate phonons, a detector, or as in the present case the coupling to leads. For the transient dynamics during a total propagation

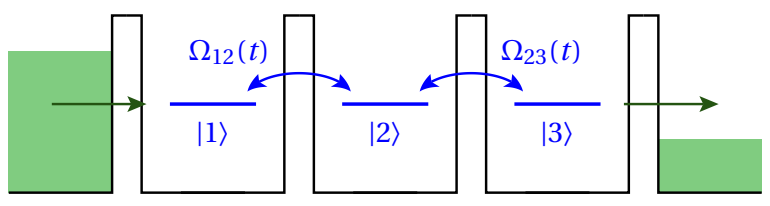

FIG. 1. Triple quantum dot in linear arrangement, where the first and the last dot are tunnel coupled to electron reservoirs with a large voltage bias applied. The CTAP protocol can be implemented by proper time-dependent gate voltages at the inter-dot barriers, such that the corresponding tunnel matrix elements $\Omega_{12}(t)$ and $\Omega_{23}(t)$ obey Eq. 2. 

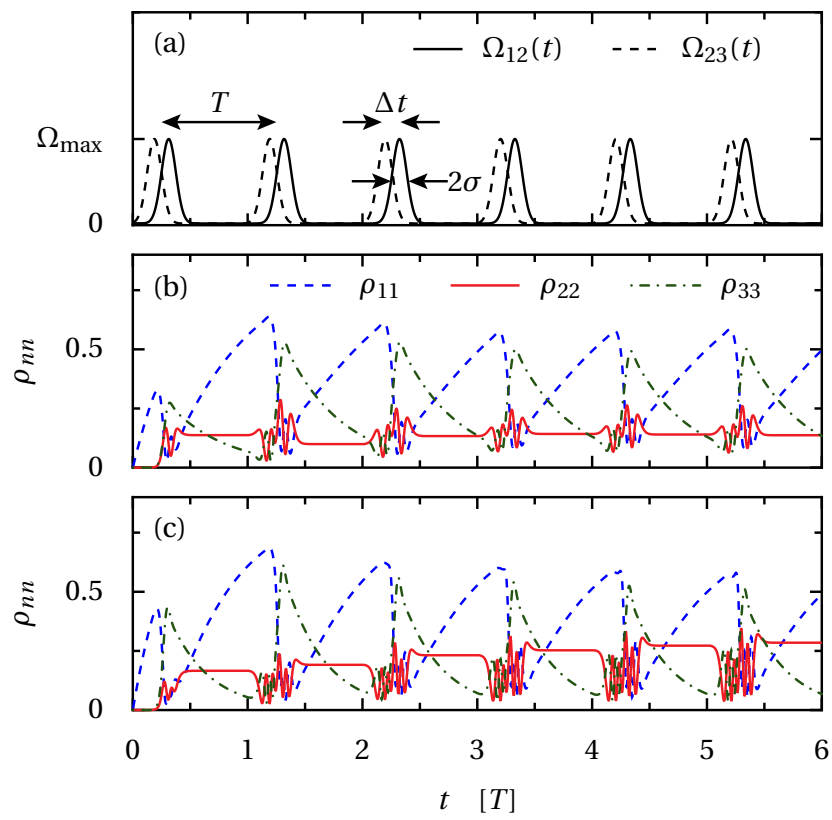

FIG. 2. (Color online) (a) Time profile of the inter-dot tunnel matrix elements forming repeated CTAP pulses. (b,c) Time evolution of the dot occupation probabilities for $\Gamma=0.5 / \Omega_{\max }$ and driving periods (b) $T=2 / \Omega_{\max }$ and (c) $T=2.9 / \Omega_{\max }$.

time $t_{\max }$ considered in Ref. [1, decoherence is tolerable for the pulse parameters $\Delta t=2 \sigma=T / 4$. Here we use $T=t_{\max }$ as pulse repetition time and focus on the steady state. An important feature of the CTAP double pulse is its "counter-intuitive" order [1, which means that the coupling $\Omega_{23}$ is active before $\Omega_{12}$. This requirement can be understood upon noticing that $\left(\Omega_{23}, 0,-\Omega_{12}\right)^{T}$ is an eigenstate of the single-particle Hamiltonian in the basis $\{|1\rangle,|2\rangle,|3\rangle\}$. Between the maxima of the double peak, this eigenstate changes from $(1,0,0)^{T}$ to $(0,0,-1)^{T}$. Thus, the adiabatic time evolution of an electron starting in dot 1 is as deserved, namely it undergoes a direct transition to dot 3 without populating dot 2 .

The leads are modeled as free electron gases with the Hamiltonian $H_{\text {leads }}=\sum_{\ell, q} \epsilon_{q} c_{\ell q}^{\dagger} c_{\ell q}$, where $c_{\ell q}^{\dagger}$ creates an electron in state $q$ of lead $\ell=L, R$. The coupling of dot 1 to the left lead is given by the tunnel Hamiltonian $H_{L}=\sum_{q} V_{L q} c_{L q}^{\dagger} c_{1}+$ H.c. and $H_{R}$ accordingly. The lead coupling is fully determined by its spectral density $\Gamma_{L}(\epsilon)=2 \pi \sum_{q}\left|V_{L q}\right|^{2} \delta\left(\epsilon-\epsilon_{q}\right)$, which we assume within a wide-band limit energy independent and equal for both leads, $\Gamma_{L}(\epsilon)=\Gamma_{R}(\epsilon) \equiv \Gamma$.

If all relevant dot energies lie within the voltage window of the two leads, electron transport becomes unidirectional. Then one can derive for the reduced triple dot density operator $\rho$ within a Bloch-Redfield approach the master equation [12]

$$
\dot{\rho}=-i\left[H_{\mathrm{TQD}}(t), \rho\right]+\Gamma \mathcal{D}\left(c_{1}^{\dagger}\right) \rho+\Gamma \mathcal{D}\left(c_{3}\right) \rho \equiv \mathcal{L}(t) \rho
$$

with the Lindblad form $\mathcal{D}(x) \rho \equiv x \rho x^{\dagger}-\frac{1}{2} x^{\dagger} x \rho-\frac{1}{2} x^{\dagger} x$.
The dissipative superoperators $\mathcal{D}\left(c_{1}^{\dagger}\right)$ and $\mathcal{D}\left(c_{3}\right)$ describe incoherent tunneling from the left lead (source) to dot 1 and from dot 3 to the right lead (drain), respectively.

Computing current noise by numerical propagation.The fluctuations of the current can be characterized by the current auto correlation function $S(\omega)$ which is typically measured at low frequencies. Therefore, we focus on the zero-frequency limit in which the correlation function relates to the variance of the transported charge according to $S \equiv S(0)=e^{2} \lim _{t \rightarrow \infty}(d / d t)\left\langle\Delta N^{2}(t)\right\rangle$ [13, 14, where $e$ is the elementary charge. The variance can be computed with the help of a density operator $R(\chi, t)$ that is augmented by counting variable $\chi$ and possesses the limit $R(\chi \rightarrow 0, t)=\rho(t)$. Its trace $\operatorname{tr} R(\chi, t)=\left\langle e^{i \chi N}\right\rangle$ is the moment generating function for the number of transported electrons $N$, i.e., $\left\langle N^{k}\right\rangle=\left.(\partial / \partial i \chi)^{k} \operatorname{tr} R\right|_{\chi=0}$. For unidirectional transport, $R$ obeys the master equation $\dot{R}(\chi, t)=\mathcal{L} R(\chi, t)+\left(e^{i \chi}-1\right) \mathcal{J} R(\chi, t)$ [15], where $\mathcal{J} R=\Gamma c_{3} R c_{3}^{\dagger}$ is the jump operator contained in the Lindblad form. Exploiting the $2 \pi$ periodicity in $\chi$, one frequently continues with a Fourier transformation which yields the so-called $N$-resolved master equation [12. Then however, a major technical problem arises, because one has to consider many additional degrees of freedom, such that a numerical solution becomes rather expensive. A more economic approach has been developed in Refs. 10, 11. By Taylor expansion up to second order, $R(\chi)=\rho+i \chi R_{1}-\frac{1}{2} \chi^{2} R_{2}$, one obtains the equations of motion $\dot{R}_{1}=\mathcal{L} R_{1}+\mathcal{J} \rho$ and $\dot{R}_{2}=\mathcal{L} R_{2}+2 \mathcal{J} R_{1}+\mathcal{J} \rho$, which we solve by numerical propagation. Then we use the fact that $\operatorname{tr} R(\chi)$ is the moment generating function, so that $(d / d t)\left\langle N^{k}\right\rangle=\operatorname{tr} \dot{R}_{k}$, by which we finally obtain the current $I=e \lim _{t \rightarrow \infty} \operatorname{tr} \dot{R}_{1}$ and the zero-frequency noise $S=e I+\lim _{t \rightarrow \infty} 2 e \operatorname{tr}\left(e \mathcal{J} R_{1}-I R_{1}\right)$. For transport in periodically time-dependent conductors, these quantities must be considered in the average over the driving period [16].

The relative noise strength is characterized by the Fano factor $F=S / e|I|$ which provides information about the transport mechanism [14]. For a Poisson process it has value one, while it becomes smaller for more regular transport. In the present context, we will speak of "significant shot noise suppression" if the Fano factor lies clearly below $1 / 2$.

Time evolution. - In order to acquire insight into the system dynamics, we time integrate master equation (3) by a Runge-Kutta method. We start with an empty triple quantum dot just before the first double pulse sets in and obtain the time evolution of the dot occupations depicted in Fig. 2(b). At the initial stage, $t \lesssim 0.1 T$, dot 1 becomes populated by an electron that tunnels in from the left lead. Between the peaks of the double pulse [cf. panel (a)], the electron essentially undergoes a direct transfer to dot 3 . As non-adiabatic correction, dot 2 acquires some occupation, which however stays clearly 


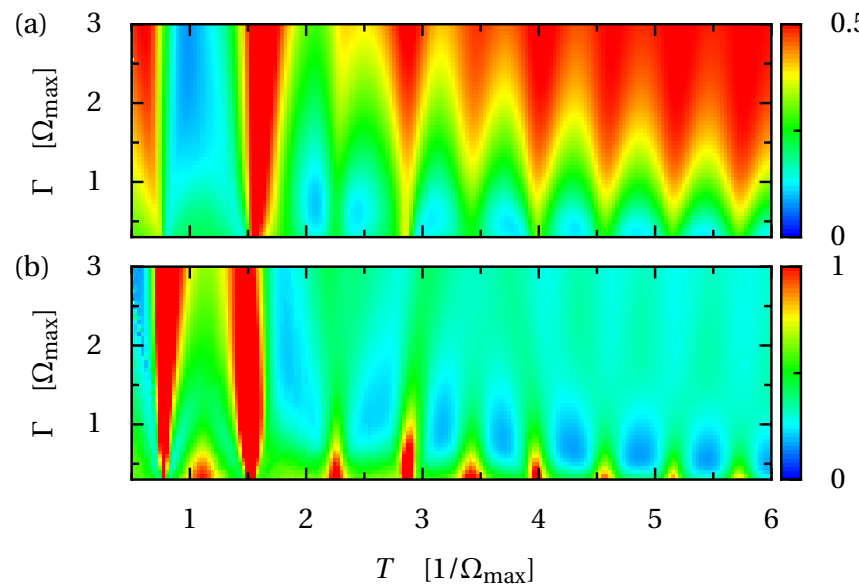

FIG. 3. (Color online) (a) Time averaged population of dot 2, $\bar{\rho}_{22}$, and (b) Fano factor $F=S / e|I|$ in the steady-state limit as function the pulse repetition time $T$ and the dot-lead coupling $\Gamma$. For graphical reasons, the data are clipped at the values 0.5 and 1 , respectively.

below the occupation of the other dots. After the double pulse, the electron tunnels further to the right lead, and a next electron may enter at dot 1 . The fast oscillation of $\rho_{22}$ when inter-dot tunneling is active can be identified as tunnel oscillation between the middle dot and one of its neighbors, because its frequency is roughly $\Omega_{\max } / 2 \pi$ [17. For certain values of the driving period $T$ [panel (c)], these oscillations become larger and may interfere constructively, so that dot 2 acquires a larger occupation.

If all tunnel matrix elements were constant in time, the population of all three dots were about equal (not shown). Thus, our transport process must be dominated by CTAP if the occupation of the middle dot is considerably smaller than the occupation of the other two dots. In the ideal case, the steady state will be such that with each double pulse, one electron is transported. This gives rise to a regular current, so that shot noise is suppressed. In the following, we analyze this current and will find that the proper course of the CTAP protocol correlates with small noise manifest in the Fano factor.

Steady state.-For computing the current noise, we need to solve simultaneously master equation (3) and the equation of motion for $R_{1}$, as discussed above. Since we are interested in the long-time behavior, we eliminate transient effects by considering the results only after all elements of the density matrix have changed by less than $0.25 \%$ with respect to the previous period. Convergence is typically reached after five to ten double pulses.

Figure 3 provides an overview of the global behavior as function of the driving period $T$ and the dot-lead coupling $\Gamma$. For the time-averaged occupation of dot 2 shown in panel (a), we distinguish three regions. First, for $T \lesssim 1.5 / \Omega_{\max }$ in which the driving is too fast, such that non-adiabatic effects play a strong role, and we can- not expect CTAP to work properly. Accordingly, the average population $\bar{\rho}_{22}$ may take any value. The corresponding Fano factor [panel (b)] is about $1 / 2$ or larger, i.e., there is no relevant shot noise suppression. Notice the similarity of the dot occupation with the one observed in Ref. [1, despite that there transient dynamics with phenomenological decoherence was considered.

For larger driving periods, we find a broad region in which the population of the middle dot is rather low, unless the dot-lead coupling $\Gamma$ becomes too large. In particular, we find islands in which both the average and the maximum of the mean occupation is as small as $\bar{\rho}_{22} \approx \rho_{22} \approx 0.1$. The plot for the Fano factor exhibits a similar structure, where both $F$ and $\bar{\rho}_{22}$ assume small values for roughly the same parameters. This provides a first hint that our generalized CTAP gives rise to regular, low-noise electron transport, in particular when $\Gamma$ is of the order $\Omega_{\max }$. Interestingly, for certain values of $T$, dot 2 becomes occupied and the Fano factor increases accordingly. This can be attributed to the mentioned tunnel oscillations, because the distance between two peaks increases linearly with the amplitude $\Omega_{\max }$.

For a more quantitative analysis, we depict in Fig. 4 the time-averaged steady-state occupation $\bar{\rho}_{22}$ and the Fano factor for two relatively small values of the dotlead coupling $\Gamma$. This demonstrates that the population of dot 2 can be as low as 0.1 , while it becomes roughly $1 / 3$ in the intermediate region. Accordingly, the Fano factor changes from a significantly sub-Poissonian value to $F \approx$ 1. The dc current [inset of Fig. 3(a)], by contrast, exhibits only minor signatures of CTAP. This underlines that it is indeed the noise which provides information about the nature of the transport process. Moreover, it reveals that for the relatively small $T$ considered, on average only each second double pulse transports an electron. This value can be improved by increasing $T$ which, however, requires a smaller dot-lead tunneling $\Gamma$.

We quantify the relation between the population $\bar{\rho}_{22}$ and the Fano factor $F$ by the correlation coefficient

$$
r=\frac{\left\langle F, \bar{\rho}_{22}\right\rangle}{\Delta F \Delta \bar{\rho}_{22}}
$$

between these quantities for $T$ in the relevant interval $\left[2 / \Omega_{\max }, 4 / \Omega_{\max }\right]$. The numerator denotes the covariance, while the denominator contains the standard deviations, such that $r=1$ for ideal correlation. The inset of Fig. $4(\mathrm{~b})$ shows that for $\Gamma \lesssim \Omega_{\max }$, the correlation is rather high, typically 0.75 or larger. Moreover, the difference between the maximum and the minimum of the Fano factor, $F_{\max }-F_{\min }$, is roughly 0.5 , i.e., sufficiently large to be discriminated in an experiment [18. For larger values of $\Gamma$, the Fano factor and the population become significantly anti-correlated. There however, $F_{\max }-F_{\min }$ is quite small and may be below the experimental resolution. Therefore we do not further discuss the regime $\Gamma \gtrsim 1.5 \Omega_{\max }$. 

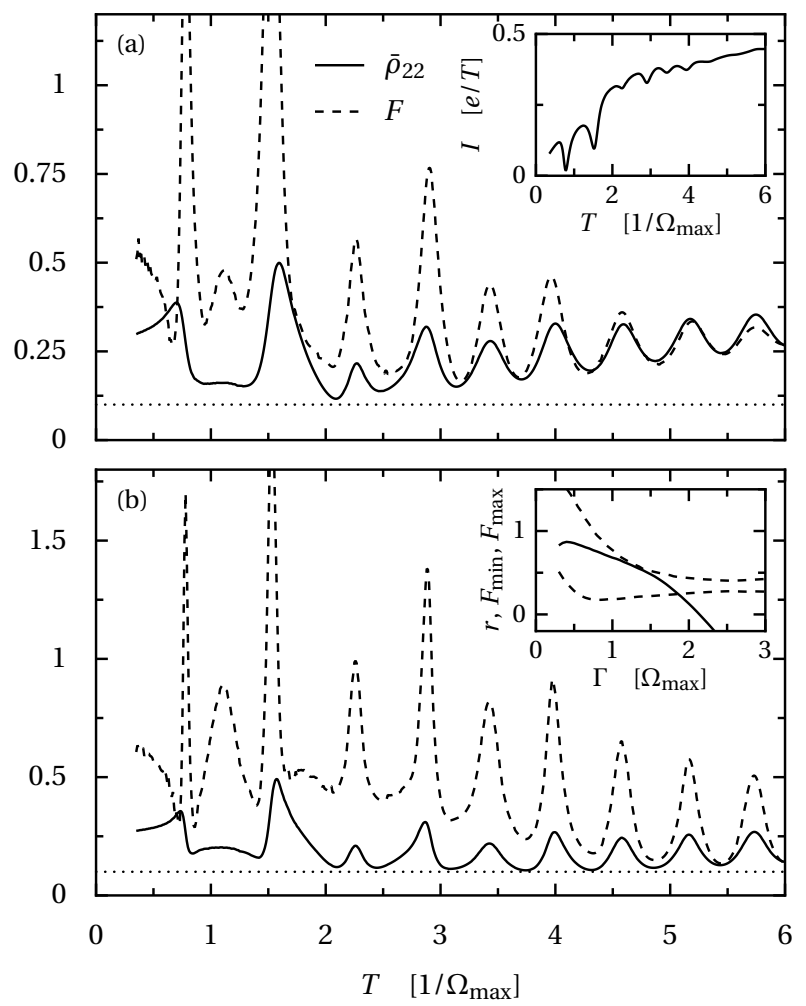

FIG. 4. Fano factor $F=S / e|I|$ and time-averaged population $\bar{\rho}_{22}$ for the dot-lead couplings (a) $\Gamma=\Omega_{\max }$ and (b) $\Gamma=0.5 \Omega_{\max }$. The horizontal dotted line marks the value 0.1 which is the minimal reminiscent population of dot 2 . Insets: (a) dc current corresponding to the main plot in units of electron per pulse. (b) Correlation coefficient (4) of the Fano factor and $\bar{\rho}_{22}$ in the range $\left[2 / \Omega_{\max }, 4 / \Omega_{\max }\right]$ (solid line) and the corresponding minimum and maximum of the Fano factor (dashed).

In a possible experimental realization of steady-state CTAP, the oscillatory dependence of the Fano factor as function of the pulse distance $T$ may serve as indicator. Most significant results are expected for $\Gamma \lesssim \Omega_{\max }$. Thus, one may use a triple quantum dot whose four barriers have similar transparency. Negative gate voltages applied to the inner barriers allow one to control the tunnel matrix elements. Since the average current consists of roughly half electron per double pulse, a current $I \approx 10 \mathrm{pA}$ requires a repetition rate of $100 \mathrm{MHz}[19$. The maximal tunnel rates should be considerably larger than that, say, by a factor 10 , which means that $\Gamma$ and $\Omega_{\max }$ should be of order $10 \mu \mathrm{eV}$.

Conclusions. - We have proposed a CTAP protocol for a triple quantum dot in transport configuration, i.e., when the first dot and the last dot are coupled to electron source and drain. It induces a steady-state transport in which electrons tunnel non-locally from the first to the last dot, practically without populating the middle dot. The noise properties of the resulting current depend on the size of the reminiscent population. The difference is manifested in the Fano factor which, thus, represents a fingerprint of the proper course of the protocol. Such indirect evidence for CTAP is particularly useful, because any direct measurement of the dot occupations would act back on the system and, thus, unavoidably influence the effect to be substantiated. A particular feature of steady-state CTAP is that it fails for certain distances between pulse pairs. Thus, upon changing this distance, one obtains a sequence of sizable shot noise suppression and shot noise enhancement, which should be measurable by standard techniques. Thus, our proposal may initiate the experimental realization of this intriguing non-local transport phenomenon.

This work was supported by the Spanish Ministry of Economy and Competitiveness through Grant No. MAT2011-24331 and by the Marie Curie Initial Training Network through Grant No. 234970.

[1] A. D. Greentree, J. H. Cole, A. R. Hamilton, and L. C. L. Hollenberg, Phys. Rev. B 70, 235317 (2004).

[2] L. Gaudreau, S. A. Studenikin, A. S. Sachrajda, P. Zawadzki, A. Kam, J. Lapointe, M. Korkusinski, and P. Hawrylak, Phys. Rev. Lett. 97, 036807 (2006).

[3] D. Schröer, A. D. Greentree, L. Gaudreau, K. Eberl, L. C. L. Hollenberg, J. P. Kotthaus, and S. Ludwig, Phys. Rev. B 76, 075306 (2007).

[4] J. Oreg, F. T. Hioe, and J. H. Eberly, Phys. Rev. A 29, 690 (1984).

[5] J. R. Kuklinski, U. Gaubatz, F. T. Hioe, and K. Bergmann, Phys. Rev. A 40, 6741 (1989).

[6] S. Gustavsson, R. Leturcq, B. Simovič, R. Schleser, T. Ihn, P. Studerus, K. Ensslin, D. C. Driscoll, and A. C. Gossard, Phys. Rev. Lett. 96, 076605 (2006).

[7] C. Fricke, F. Hohls, W. Wegscheider, and R. J. Haug, Phys. Rev. B 76, 155307 (2007).

[8] J. Rech and S. Kehrein, Phys. Rev. Lett. 106, 136808 (2011).

[9] I. Kamleitner, J. Cresser, and J. Twamley, Phys. Rev. A 77, 032331 (2008).

[10] F. J. Kaiser and S. Kohler, Ann. Phys. (Leipzig) 16, 702 (2007).

[11] R. Sánchez, S. Kohler, and G. Platero, New J. Phys. 10, 115013 (2008).

[12] S. A. Gurvitz and Ya. S. Prager, Phys. Rev. B 53, 15932 (1996).

[13] D. K. C. MacDonald, Rep. Prog. Phys. 12, 56 (1949).

[14] Ya. M. Blanter and M. Büttiker, Phys. Rep. 336, 1 (2000).

[15] C. Flindt, T. Novotný, and A.-P. Jauho, Phys. Rev. B 70, 205334 (2004).

[16] S. Kohler, J. Lehmann, and P. Hänggi, Phys. Rep. 406, 379 (2005).

[17] T. Brandes and T. Vorrath, Phys. Rev. B 66, 075341 (2002).

[18] P. Barthold, F. Hohls, N. Maire, K. Pierz, and R. J. Haug, Phys. Rev. Lett. 96, 246804 (2006).

[19] T. Hayashi, T. Fujisawa, H. D. Cheong, Y. H. Jeong, and Y. Hirayama, Phys. Rev. Lett. 91, 226804 (2003). 